\title{
Graffitis - le « cadre » du photographe
}

\section{Gustave Deghilage}

\section{(2) OpenEdition}

\section{Journals}

Édition électronique

URL : https://journals.openedition.org/clo/2309

DOI : $10.4000 /$ clo.2309

ISSN : 2266-1816

\section{Éditeur}

INALCO

\section{Édition imprimée}

Date de publication : 31 décembre 2015

ISBN : 9782858312276

ISSN : 0396-891X

\section{Référence électronique}

Gustave Deghilage, "Graffitis - le « cadre » du photographe », Cahiers de littérature orale [En ligne], 77-78 | 2015, mis en ligne le 10 mai 2016, consulté le 30 juin 2021. URL : http:// journals.openedition.org/clo/2309; DOI : https://doi.org/10.4000/clo.2309

\section{(ब) (1) $\odot$}

Cahiers de littérature orale est mis à disposition selon les termes de la Licence Creative Commons Attribution - Pas d'Utilisation Commerciale 4.0 International. 


\title{
Graffitis - le « cadre » du photographe
}

\author{
Gustave Deghilage
}

Les graffitis sont fascinants, ne trouvez-vous pas ? Ils sont la voix silencieuse de ceux qui pensent n'avoir que les murs de nos villes pour tenter d'exister, pour exprimer leurs doutes, leur colère ou leur révolte, la tendresse, l'amour, mais aussi la haine de l'autre ou de soi-même, voire de la société dans laquelle ils vivent et qu'ils considèrent parfois comme un système hostile qui ne leur laisse qu'une place congrue.

Or écrire sur un mur n'est jamais anodin. C'est d'abord une transgression : transgression de la propriété privée ou de l'espace public que l'on « souille » (comme cela est généralement ressenti), mais transgression aussi dans le discours, par la contestation qu'il exprime, l'insulte qu' il contient ou par l'exposition de soi au-delà de ce que tolèrent les normes sociales. Ainsi dans ces graffitis on crie son mal-être, on dit souvent ce qui ne peut être dit en face à face. Car le graffiti protège autant qu'il expose : je m'expose, je m'exprime, mais je ne dis pas qui je suis.

Le graffiti joue, à différents niveaux, avec les codes et se déjoue des interdits. Il est une prise de risque donc, qui implique une urgence à laquelle peut venir répondre tout aussi bien le contenu du discours (le signifié) que le geste qui le pose et la montée d'adrénaline qu'il procure. À chaque inscription, il y a ainsi une raison propre, que l'on peut imaginer, fantasmer. Mais là n'est pas mon propos. Tout au plus peut-on présumer que le message - ou l'acte qui le pose - répond à un impératif. Car sa mise en œuvre demande une préméditation, et parce qu'en imprimant sa marque, il dégrade - aux yeux de la loi - la propriété ou l'espace public. Parce qu'il est pénalement condamnable, le discours acquiert un poids : il n'est jamais complètement futile et cet acte de transgression sépare l'essentiel de l'anecdotique. Car graffer, bien souvent, c'est exister. C'est une manière, non 
pas de se positionner en dehors de la société, mais de s'y inscrire, même si c'est en marge, même si c'est hors du cadre. Même si c'est dans... l'anonymat ${ }^{1}$.

Les murs sont ce qui sépare le privé du public. Ils protègent l'intime, autant des intrusions que des regards indiscrets. Ils sont solides, inébranlables, et la façade - qu'elle soit fleurie, décrépite ou rajeunie, aux lignes banales, délicieusement désuètes ou résolument futuristes - est la seule part de notre intimité que nous offrons, bon an, mal an, aux regards du tout-venant. Une façade est apparence, et ainsi potentiellement trompeuse. Mais, quoi qu'il en soit, elle demeure, par nature, la face visible de l'intime.

Alors quand un cri du cœur fleurit en ces lieux, il est tentant de s'interroger sur ce qu'il peut signifier : inscrire, précisément, sur cette carapace urbaine, le tropplein de son âme est un acte, en apparence du moins, étrangement paradoxal, car le mur est précisément ce qui devrait protéger notre vie intime.

Il y a, dans ce jeu d'écritures, comme une bouteille que l'on jette à la mer, le besoin de dire un essentiel, de revendiquer son ressenti, de le faire reconnaitre en le « criant » à la face du monde. Mais il y a aussi une indéniable pudeur, un jeu de cache-cache et, si certains messages sont identifiables, ils ne le sont bien souvent que des seuls initiés. On n'écrit ainsi pas sur le mur de sa propre maison. On écrit sur le mur d'un autre, mais sans que le message lui soit pour autant directement adressé. Celui-ci n'est d'ailleurs que rarement destiné à une personne en particulier : il prend, par nature, le passant à témoin. Une façon peut-être de déléguer sa parole à d'autres, de la mettre à distance en tout cas, de rester anonyme. Dire ce qui nous est essentiel tout en se préservant est sans doute aussi une manière de se construire une part de dignité. Comme si le « graffeur » nous disait en quelque sorte : « Non, je ne veux pas être vu, je veux produire du sens. Et je veux seulement que ce que j'ai à dire soit vu. »

Les formes de ces inscriptions, comme les messages qu'elles véhiculent, sont multiples. Esthétiques ou non, figuratives ou abstraites, mots ou silences, sympathiques et drôles, voire poétiques ou franchement vulgaires, elles sont autant de messages laissés là, à la vue de tous. Elles interpellent forcément le regard, nous questionnent, et nous amènent à prendre parti. Nous pouvons les ignorer, passer notre chemin. Mais pour qui sait regarder, elles sont souvent un enrichissement, en ce sens au moins qu'elles sont les signes - ou les symptômes - de ce qui se passe dans le microcosme urbain. Elles participent de notre connaissance, elles nous aident à comprendre, elles sont des messages à ne pas négliger : à nous d'en tirer des moyens d'agir.

1. Voir à ce propos Street View, l'excellent film de Virginie Grandhomme, CNRS Images, 2013. 
En écrivant sur un mur, le « graffeur » s'approprie et se construit un espace de parole, qui n'est originellement pas destiné à cet usage (hormis pour les publicités, messages institutionnalisés et mercantiles). Cet espace lui permet de diffuser son discours tout en restant anonyme. Mais aussitôt écrit, il en perd le contrôle et le message se détache de son auteur pour commencer sa vie, pour ainsi dire autonome.

L'écriture, en effet, inscrit le discours dans un espace temporel indéfini (qui peut être très court, mais potentiellement « infiniment » long, songez à Lascaux) et l'expose dans un espace topographique, certes très variable, mais qui possède une propriété particulière qui en fait sa raison d'être : il est public. Elle lui confère ainsi une puissance que ne possède pas l'oral : jusqu'à ce qu'il soit effacé, il s'offre et s'impose au passant, multipliant à l'envi les témoins potentiels de cette parole : visible de tous, il n'y a pas de tri parmi les destinataires, autre que le fait de passer par là. Le graffiti est donc une parole publique au plein sens du terme.

Comme il est souvent concis, il ne faut qu'un seul coup d'œil pour l'appréhender. Le regard s'y arrête. Ou non. Mais dès qu'il s'est posé, le message s'impose à nous. Il nous amuse ou il nous choque ; il nous émerveille ou nous rebute. Il peut aussi nous laisser indifférents.

J'aime découvrir ces graffitis au gré de mes cheminements en ville, dans ma ville ou lors de mes voyages plus lointains, et j'éprouve - il est vrai - un certain plaisir à photographier ces traces vivantes et souvent fugaces des pensées, des aspirations, des rires ou « des coups de gueule » des gens de la rue. J'aime les donner à voir, les transmettre à mon tour un peu plus loin, comme on passe un témoin : une manière pour moi de prolonger un questionnement, un enthousiasme ou une révolte.

Je ne suis pas pour autant d'accord avec tous les messages que je relaie - je n'ai pas à l'être, on ne me le demande d'ailleurs pas. Je donne à voir ce qui m'a touché, interpelé ou révolté ; je donne à voir la poésie, la subtilité d'une formule ou la beauté d'un trait, mais aussi parfois ce que je considère comme la bêtise crasse de mes congénères (en cela, je me démarque d'une démarche purement analytique ou scientifique, puisque je me permets la subjectivité).

Le fait de présenter ainsi des messages hors de leur « milieu naturel » leur donne alors un sens différent qui est de l'ordre métadiscursif, même s'il est rare que j'y ajoute un commentaire (hormis celui que vous lisez ici, bien entendu, mais qui n'est pas constitutif de ma démarche habituelle et qui, volontairement, ne concerne pas spécifiquement l'image que j'ai choisi de vous montrer).

Comme tout travail de photographie, ma démarche est esthétisante, c'est-àdire qu'elle cherche à mettre à distance en même temps qu'elle révèle, rend visible et potentiellement digne d'intérêt l'anecdotique et le quotidien, tout ce qui en 
apparence peut paraître insignifiant : je me veux témoin de l'écume qu'engendre notre petit monde humain...

Si je me demande pourquoi un inconnu a ressenti le besoin de déposer son message sur un mur, ceux qui regardent mes images se demanderont peut-être (c'est du moins mon souhait) pourquoi j'ai choisi de vous faire découvrir ce message. Et cela est en soi très différent : la question ne porte plus sur le message mais sur le regard qui le fixe.

Car j'instaure immanquablement une médiation : celle de mon regard, qui s'est arrêté, celle de ma pensée, de ma sensibilité, de mes émotions; de tout ce qui m'a fait revenir sur un lieu, muni de mon appareil photo. Je donne à voir, je partage, je prolonge. Et si ma citation a apparence d'objectivité, elle ne l'est pourtant jamais totalement. Car en donnant à voir, je propose un point de vue : l'image porte ainsi en elle l'empreinte de ma subjectivité, plus ou moins explicite, plus ou moins décelée. Comme finalement, le travail de l'anthropologue ou celui de l'ethnographe est de part en part transpercé par la subjectivité du chercheur. Tout n'est, dans les sciences humaines comme en photographie - c'est du moins ce que je pense - qu'une question de cadrage.

Les graffitis sont fascinants, disais-je. C'est pourquoi j'ai choisi de m'en emparer et de les exposer à travers mes photographies ${ }^{2}$. Ces photos sont une manière pour moi de répercuter leur message. Je ne rencontrerai vraisemblablement jamais celle qui a écrit « je serais restée dans le ventre de ma maman », mais, par la diffusion de la photo sur différents supports, j'en amène d'autres à être témoins à leur tour. Cette exposition sublime l'objet et en détourne la fonction : ce graffiti illustre désormais la couverture d'un livre; on y voit certes encore la douleur qu'il exprime (feinte, sincère ou ironique, je vous en laisse juge), mais on découvre aussi une beauté à ce message... Le mur qui le portait a été repeint depuis, mais son discours demeure, et cet ouvrage s'en fait à son tour le vecteur. Et ici personne ne pourra l'effacer...

2. D'autres sont visibles ici : https://www.flickr.com/photos/degust/collections/ 